\title{
Persistence of Denosumab Therapy among Patients with Osteoporosis
}

\author{
Kazuyoshi Kobayashi, Kei Ando, Masaaki Machino, Masayoshi Morozumi, Shunsuke Kanbara, \\ Sadayuki Ito, Taro Inoue, Hidetoshi Yamaguchi, Naoki Ishiguro, Shiro Imagama \\ Department of Orthopedic Surgery, Nagoya University Graduate School of Medicine, Nagoya, Japan
}

\begin{abstract}
Study Design: Retrospective study in a single center.
Purpose: To examine denosumab persistence in patients of different ages with severe osteoporosis in Japan.

Overview of Literature: Denosumab is an antibody drug used for the treatment of osteoporosis. It is mainly used in patients with severe osteoporosis who might have high motivation for treatment, and the need for only semi-annual subcutaneous injection might improve the continuation rate. However, no English-language articles have reported on denosumab persistence in the Japanese population, including young people, despite the importance of this issue in a super-aging society.

Methods: The subjects started treatment with subcutaneous denosumab in our department from July 2013 until December 2017. Persistence rates were calculated using Kaplan-Meier curves. Patients were defined as "persistent" or "non-persistent" according to the use of therapy after 60 months.

Results: The study included 101 patients (84 females) with a median follow-up period of 23.6 \pm 14.2 months. The persistence rate declined to $85.3 \%, 78.3 \%, 74.1 \%, 71.3 \%$, and $69.3 \%$ at $12,24,36,48$, and 60 months, respectively. Age at the initiation of denosumab therapy differed significantly between non-persistent $(n=31)$ and persistent $(n=70)$ patients ( 81.3 vs. 72.8 years, $p<0.01)$. Persistence was significantly lower in patients aged $\geq 80$ years than in those aged $<60$ and $60-79$ years (both $p<0.01$ ). The reasons for non-persistence of denosumab therapy were transfer to another hospital $(n=13)$, interruption of outpatient visits $(n=11)$, dental treatment $(n=4)$, adverse events ( $n=2)$, and patient request $(n=1)$.

Conclusions: Persistence was significantly lower in patients aged $\geq 80$ years than in patients of other ages, and strategies promoting persistence are needed for these elderly patients.
\end{abstract}

Keywords: Denosumab; Japan; Osteoporosis; Retrospective study; Persistence

\section{Introduction}

Osteoporosis is a chronic and progressive metabolic skeletal disease characterized by low bone mass and deterioration of the microarchitecture of bone tissue, and it is associated with pain and disability $[1,2]$. Fractures due to osteoporosis are a major cause of morbidity owing to associated pain and immobility, and these fractures increase mortality, with hip fractures associated with half of the deaths attributable to osteoporosis [3]. Fragility fractures

Received Jul 16, 2019; Revised Sep 2, 2019; Accepted Sep 19, 2019

Corresponding author: Shiro Imagama

Department of Orthopedic Surgery, Nagoya University Graduate School of Medicine, 65 Tsurumai Showa-ward, Aichi 466-8550, Japan

Tel: +81-52-741-2111, Fax: +81-52-744-2260, E-mail: imagama@med.nagoya-u.ac.jp 
also place a considerable burden on healthcare resources.

Several pharmacological treatments for osteoporosis have been developed in the last 2 decades, and these were found to be effective for reducing the risk of fragility fractures in controlled clinical trials [4,5]. As osteoporosis is a chronic condition that requires long-term treatment, poor persistence with and adherence to medication are key issues. To achieve efficacy and a successful treatment outcome in a real-world setting, therapies proven to be effective in clinical trials should be taken at the prescribed dose (adherence) and should be continued over the required course (persistence) [6]. Non-persistence and nonadherence with osteoporosis therapy are associated with a high fracture risk of $30 \%-40 \%$ [7,8]. Additionally, recent evaluations of fragility fractures have shown that lack of efficacy because of poor therapy persistence and/or adherence results in high economic cost and reduces healthrelated quality of life of patients $[9,10]$. This is a major public health issue, and ensuring adherence and persistence of osteoporosis treatment is particularly challenging for healthcare professionals.

Denosumab (Prolia; Amgen, Thousand Oaks, CA, USA) is a fully human monoclonal antibody that selectively targets the RANK ligand and is an alternative antiresorptive treatment for osteoporosis [11]. It is the first biological agent developed as an anti-osteoporosis drug, is administered by subcutaneous injection every 6 months, and has been authorized for the treatment of osteoporosis in Japan since 2013 [12]. In clinical trials, denosumab has been shown to be well tolerated, increase bone mineral density (BMD), and greatly reduce fracture risk [12]. Denosumab administered every 6 months in patients with severe osteoporosis was highly effective for decreasing fracture risk, with a $68 \%$ reduction in the incidence of new radiographic vertebral fractures in 3 years and a similar $69 \%$ reduction in the incidence of clinical vertebral fractures [13-15]. Therefore, denosumab is mainly used for patients with severe osteoporosis who might have high motivation for treatment, and the need for only semi-annual subcutaneous injection might improve the continuation rate. Regarding denosumab persistence, Silverman et al. [16] found a 12 -month discontinuation rate of $18 \%$ and $\mathrm{Mi}$ gliaccio et al. [17] reported persistence rates of $83 \%, 69 \%$, and $62 \%$ at 12,18 , and 24 months, respectively. However, no English-language articles have reported on denosumab persistence in the Japanese population, including young people, despite the importance of this issue in a super- aging society. Therefore, the present study was designed to examine denosumab persistence for the treatment of osteoporosis among different age groups in a routine clinical setting in Japan.

\section{Materials and Methods}

\section{Participants}

The subjects were patients who started treatment with subcutaneous denosumab (Prolia) at $60 \mathrm{mg}$ every 6 months in our department from July 2013 until December 2017 (data collection deadline). The inclusion criteria were use of denosumab for primary osteoporosis, bone density $\leq 70 \%$ of young adult mean (YAM), history of proximal femoral fracture or vertebral fracture, and other fragile fractures with bone density $\leq 80 \%$ of YAM. Patients with secondary osteoporosis due to steroid use were also included in the study. At the beginning of denosumab treatment, patients were provided instructions on the drug by the referring orthopedic specialist. All patients received dental treatment before receiving denosumab. The following information was collected: age, period of use, year of initiation, and cause of non-persistence. Persistence has been defined as the duration from initiation to discontinuation of therapy [18]. In the present study, persistence was evaluated as a dichotomous variable measured at the end of a predefined period, with patients defined as "persistent" or "non-persistent." Persistence rates were calculated for all eligible patients [17], and KaplanMeier curves were used to depict persistence over time in each age group. This study was approved by the Nagoya University Institutional Review Board (IRB approval no., 2018-377), and informed consent was obtained from all individual participants included in the study.

\section{Statistical analysis}

Demographic and clinical characteristics of the subjects are summarized in the results section. The persistence rate was estimated using Kaplan-Meier analysis. Continuous variables are expressed as mean \pm standard deviation, and categorical variables are expressed as absolute value and percentage. Differences between two groups were analyzed using Student $t$-test, and differences among three groups were analyzed using the Kruskal-Wallis test. All statistical analyses were performed using IBM SPSS ver. 
22.0 (IBM Corp., Armonk, NY, USA). A p-value <0.05 was considered significant in all analyses.

\section{Results}

In the study period, we identified 101 patients (84 females [83\%]) who were treated with denosumab. The basic characteristics of the cohort are shown in Table 1. The median follow-up period was 23.6 \pm 14.2 months (range, 8.1-66.2 months). The age distribution of the patients is shown in Fig. 1. Among the patients, three were $<40$ years old and two were $>90$ years old, indicating a wide age range. There were 31 non-persistent patients, including 4 (20\%), 9 (19\%), and $18(53 \%)$ aged $<60,60-79$, and $\geq 80$ years, respectively. The reasons for non-persistence of denosumab are shown in Table 2. The most common cause was transfer to another hospital $(n=13)$, followed by interruption of

Table 1. Characteristics of the study population $(n=101)$

\begin{tabular}{|cc|}
\hline Characteristic & Value \\
\hline Follow-up period (mo) ${ }^{\text {a) }}$ & $23.6 \pm 14.2$ \\
\hline Age $(\mathrm{yr})^{\mathrm{b})}$ & $72.3 \pm 16.8$ \\
\hline Gender (female) & $84(83)$ \\
\hline Year of initiation & $7(7)$ \\
\hline 2013 & $15(15)$ \\
\hline 2014 & $20(20)$ \\
\hline 2015 & $27(27)$ \\
\hline 2016 & $32(32)$ \\
\hline 2017 & $31(31)$ \\
\hline Non-persistence & \\
\hline
\end{tabular}

Values are presented as mean \pm standard deviation or number (\%).

a) Time until censoring or non-persistence. ${ }^{\text {b) }}$ Age of patient at initiation of denosumab treatment. outpatient visits $(n=11)$, dental treatment $(n=4)$, adverse events $(n=2)$, and patient request $(n=1)$. The adverse events were fatigue and nausea.

During the study period, the overall persistence rate at 60 months was 70\% (70 patients). Kaplan-Meier analysis showed a slow decline of persistence to $85.3 \%, 78.38 \%$, $74.1 \%, 71.3 \%$, and $69.3 \%$ at $12,24,36,48$, and 60 months

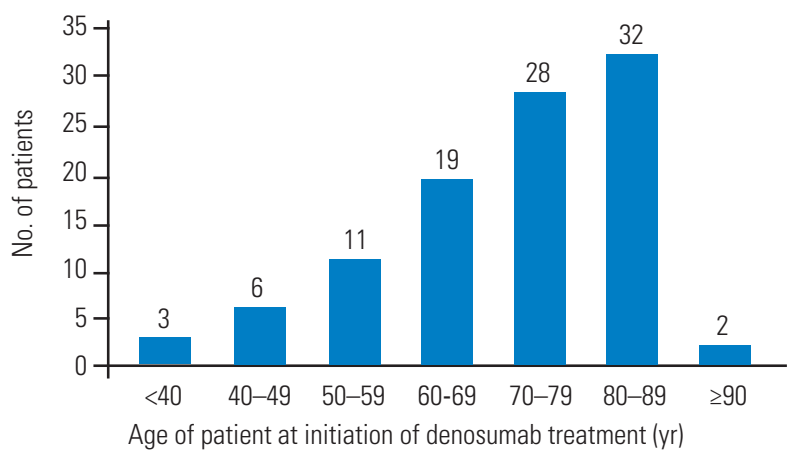

Fig. 1. Age distribution of the patients $(n=101)$.

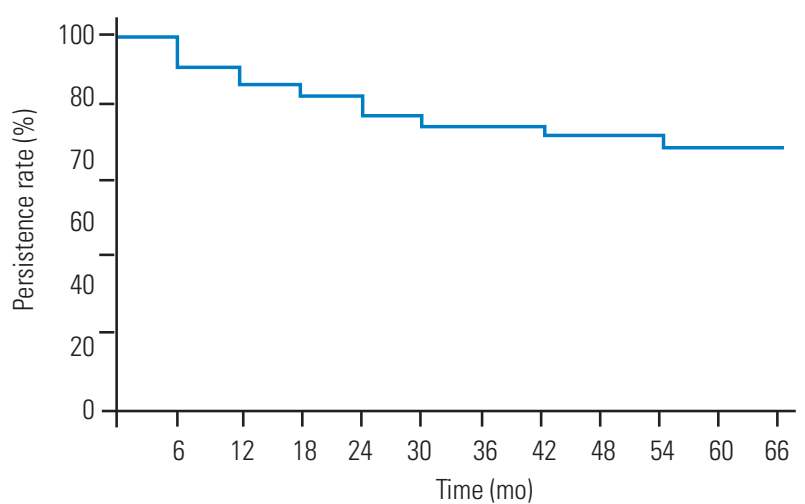

Fig. 2. Kaplan-Meier plot showing the probability of all patients continuing with denosumab treatment at a given time point. The rate dropped to $85.3 \%$, $78.3 \%, 74.1 \%, 71.3 \%$, and $69.3 \%$ at $12,24,36,48$, and 60 months of followup, respectively.

Table 2. Cause of non-persistence in different age groups $(n=31)$

\begin{tabular}{lcccc} 
& & & Age (yr) \\
Cause of non-persistence & Total & $<60$ & $60-79$ & 7 \\
\hline Transfer to another hospital & $13(40)$ & 2 & 4 & 2 \\
\hline Interruption of outpatient visits ${ }^{\text {a) }}$ & $11(34)$ & 1 & 1 & 1 \\
\hline Interruption due to dental treatment & $4(13)$ & 0 & 1 & 1 \\
\hline Adverse event & $2(6)$ & 0 & 9 & 18 \\
\hline Patient's request & $1(3)$ & 4 & 9 \\
\hline Total & 31 & & 1 \\
\hline
\end{tabular}

Values are presented as number (\%) or number.

${ }^{a}$ The rate of interruption of outpatient visits was significantly greater in patients aged $\geq 80 \mathrm{yr}(p<0.05$ by Kruskal-Wallis test). 


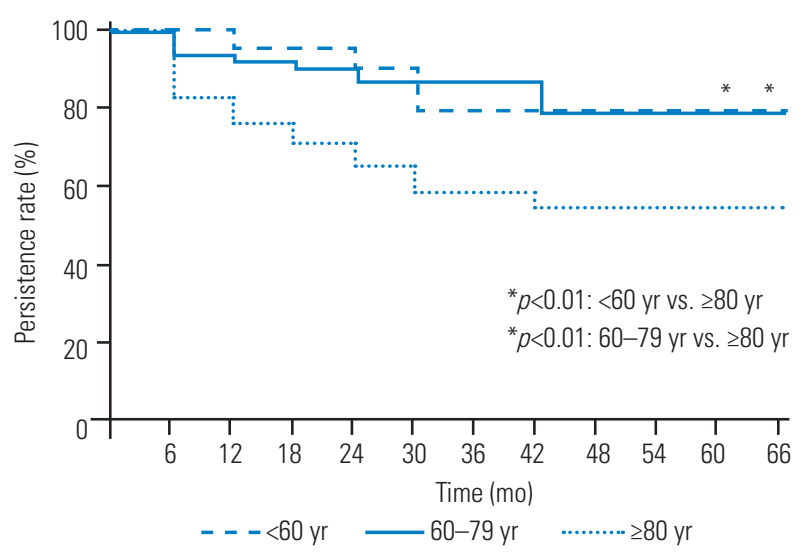

Fig. 3. Kaplan-Meier plot showing the probability of patients in each age group continuing with denosumab treatment at a given time point. Persistence is significantly lower in patients aged $\geq 80$ years than in those aged $<60$ and $60-79$ years (both $p<0.01$ ).

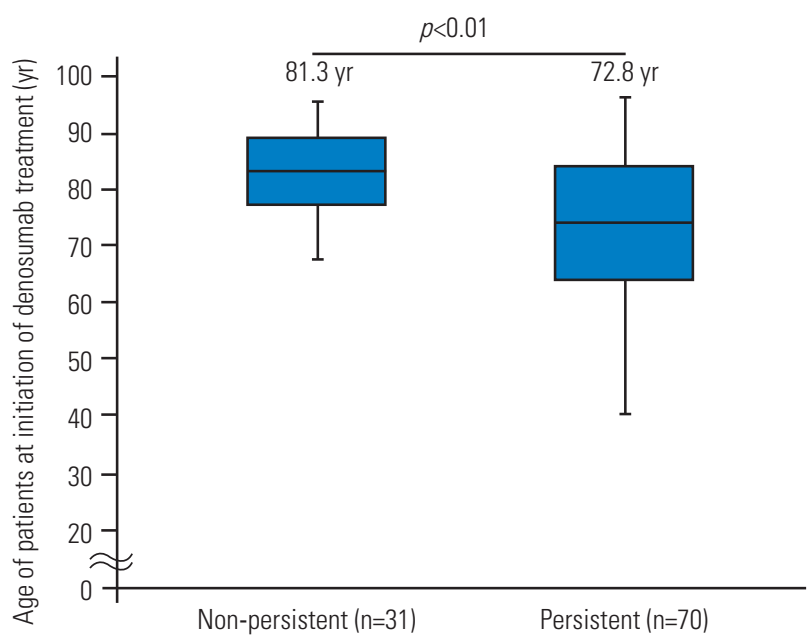

Fig. 4. There is a significant difference in age at the initiation of denosumab treatment between non-persistent $(\mathrm{n}=31)$ and persistent $(\mathrm{n}=70)$ patients $(81.3$ vs. 72.8 years, $p<0.01)$.

of follow-up, respectively (Fig. 2). With regard to age, persistence was significantly lower in patients aged $\geq 80$ years than in those aged $<60$ and $60-79$ years (both $p<0.01$ ) (Fig. 3). Additionally, there was a significant difference in age at the initiation of denosumab treatment between non-persistent $(\mathrm{n}=31)$ and persistent $(\mathrm{n}=77)$ patients ( 81.3 versus 72.8 years, $p<0.01)$ (Fig. 4$)$.

\section{Discussion}

In this study, we analyzed denosumab persistence in patients with osteoporosis. Information was collected from an orthopedic department at a single institution in Japan, and it included findings for young and old patients. Patients treated with denosumab are commonly motivated to receive osteoporosis treatment and thus are more adherent to this treatment [19]. Clinical trials have shown high denosumab persistence $[15,20]$. In a multicenter prospective study involving 935 postmenopausal women treated for osteoporosis in US and Canadian community practice, Silverman et al. [16] found a 12-month discontinuation rate of $18 \%$, whereas Migliaccio et al. [17] reported persistence rates of $83 \%, 69 \%$, and $62 \%$ at 12,18 , and 24 months, respectively. In a retrospective study by Karlsson et al. [21] using real-world data from the Swedish Prescribed Drug Register $(n=2,315)$, the denosumab persistence rate was $83 \%$ at 12 months and $62 \%$ at 24 months. It should be noted that these studies mainly included postmenopausal women, and few studies included young people.

Persistence is a key factor for the successful management of chronic disorders, and long dosing intervals are thought to contribute to better treatment adherence and persistence [22]. Therefore, understanding the factors that influence patient perceptions of osteoporosis treatment might allow improved education to increase persistence of drugs, including anti-osteoporotic drugs administered by injection, such as teriparatide and denosumab. Adequate patient monitoring, visit frequency, pharmacological schedule, and patient motivation can play important roles in enhancing persistence and increasing the effectiveness of anti-osteoporotic therapy [7].

We noted high rates of denosumab persistence of $85.3 \%$ at 12 months and $69.3 \%$ at 60 months. Administration was discontinued for dental treatment in four cases. Another reason for non-persistence was transfer to another hospital to visit a doctor in the local neighborhood. In particular, our series included many patients aged over 90 years, and it was difficult for many of them to visit our institution. These patients preferred local medical treatment, and some who had high motivation for treatment and who had been adherent to denosumab might have continued with the treatment. Additionally, some patients discontinued visiting the outpatient clinic, and this was partly because of death in elderly patients with short life expectancy. Consideration of these factors would have increased the persistence rate. The two adverse events of fatigue and nausea were also associated with non-persistence. Based on our findings, the continuation rate might be increased by (1) performing dental examination before denosumab administration, (2) pre-prescribing drugs to prevent nausea as a complication, (3) providing patients with a good explanation of the necessity and efficacy of 
denosumab, and (4) providing assistance to families, especially with regard to elderly patients.

Among anti-osteoporosis drugs, denosumab, which selectively targets the RANK ligand, might be particularly useful in elderly people, considering its high efficacy for decreasing fracture risk and its simplicity of use with only a semi-annual subcutaneous injection. Because of aging of the society, it is likely that an increasing number of people aged over 90 years will receive denosumab. We found it difficult to follow such patients because our study was performed in a university hospital rather than a facility supporting home care. However, we suggest that patients aged over 90 years can take denosumab with sufficient compliance through follow-up by their primary doctors.

The present study has some limitations. First, the sample size was small in this retrospective series. Second, a follow-up BMD test could not be performed in all cases after denosumab administration, and thus, the therapeutic effect was not evaluated. Third, the study focused only on denosumab, and there was no control group receiving a different medication. Nevertheless, the study included male and female individuals of all ages, and data were collected for young and old patients. To our knowledge, this is the first English report to describe the persistence of denosumab and the causes of denosumab discontinuation, with a focus on Japan. Studies using prescriptionbased databases have several well-known limitations that might result in underestimation of persistence; however, in Japan, denosumab is injected subcutaneously by medical staff in a hospital, making the procedure and followup data more reliable.

\section{Conclusions}

We found a high rate of persistence with denosumab therapy at a single institution in Japan. Persistence was significantly lower in patients aged $\geq 80$ years than in patients of other ages, and strategies promoting persistence are needed for these elderly patients. Our findings might be indicators of future difficulties with therapy in a superaging population.

\section{Conflict of Interest}

No potential conflict of interest relevant to this article was reported.

\section{Author Contributions}

Analyzed data and wrote the manuscript: Kazuyoshi Kobayashi; designed the study and collected data: Kei Ando, Masaaki Machino, Masayoshi Morozumi, Shunsuke Kanbara, Sadayuki Ito, Taro Inoue, Hidetoshi Yamaguchi, Naoki Ishiguro, Shiro Imagama.

\section{References}

1. Svedbom A, Hernlund E, Ivergard M, et al. Osteoporosis in the European Union: a compendium of country-specific reports. Arch Osteoporos 2013;8:137.

2. Penning-van Beest FJ, Erkens JA, Olson M, Herings RM. Loss of treatment benefit due to low compliance with bisphosphonate therapy. Osteoporos Int 2008;19:511-7.

3. Hernlund E, Svedbom A, Ivergard M, et al. Osteoporosis in the European Union: medical management, epidemiology and economic burden: a report prepared in collaboration with the International Osteoporosis Foundation (IOF) and the European Federation of Pharmaceutical Industry Associations (EFPIA). Arch Osteoporos 2013;8:136.

4. McClung M, Harris ST, Miller PD, et al. Bisphosphonate therapy for osteoporosis: benefits, risks, and drug holiday. Am J Med 2013;126:13-20.

5. Vescini F, Attanasio R, Balestrieri A, et al. Italian association of clinical endocrinologists (AME) position statement: drug therapy of osteoporosis. J Endocrinol Invest 2016;39:807-34.

6. Kanis JA, McCloskey EV, Johansson H, et al. European guidance for the diagnosis and management of osteoporosis in postmenopausal women. Osteoporos Int 2013;24:23-57.

7. Ross S, Samuels E, Gairy K, Iqbal S, Badamgarav E, Siris E. A meta-analysis of osteoporotic fracture risk with medication nonadherence. Value Health 2011;14:571-81.

8. Hiligsmann M, Gathon HJ, Bruyere O, Ethgen O, Rabenda V, Reginster JY. Cost-effectiveness of osteoporosis screening followed by treatment: the impact of medication adherence. Value Health 2010;13:394401.

9. Reginster JY. Adherence and persistence: impact on outcomes and health care resources. Bone 2006;38(2 Suppl 2):S18-21. 
10. Carr AJ, Thompson PW, Cooper C. Factors associated with adherence and persistence to bisphosphonate therapy in osteoporosis: a cross-sectional survey. Osteoporos Int 2006;17:1638-44.

11. Bekker PJ, Holloway DL, Rasmussen AS, et al. A single-dose placebo-controlled study of AMG 162, a fully human monoclonal antibody to RANKL, in postmenopausal women. J Bone Miner Res 2004;19:105966.

12. Cummings SR, San Martin J, McClung MR, et al. Denosumab for prevention of fractures in postmenopausal women with osteoporosis. N Engl J Med 2009;361:756-65.

13. McClung MR, Lewiecki EM, Cohen SB, et al. Denosumab in postmenopausal women with low bone mineral density. N Engl J Med 2006;354:821-31.

14. Lewiecki EM, Miller PD, McClung MR, et al. Twoyear treatment with denosumab (AMG 162) in a randomized phase 2 study of postmenopausal women with low BMD. J Bone Miner Res 2007;22:1832-41.

15. Brown JP, Prince RL, Deal C, et al. Comparison of the effect of denosumab and alendronate on BMD and biochemical markers of bone turnover in postmenopausal women with low bone mass: a randomized, blinded, phase 3 trial. J Bone Miner Res 2009;24:15361.

16. Silverman SL, Siris E, Kendler DL, et al. Persistence at 12 months with denosumab in postmenopausal women with osteoporosis: interim results from a prospective observational study. Osteoporos Int 2015;26:361-72.

17. Migliaccio S, Francomano D, Romagnoli E, et al. Persistence with denosumab therapy in women affected by osteoporosis with fragility fractures: a multicenter observational real practice study in Italy. J Endocrinol Invest 2017;40:1321-6.

18. Cramer JA, Roy A, Burrell A, et al. Medication compliance and persistence: terminology and definitions. Value Health 2008;11:44-7.

19. Fuksa L, Vytrisalova M. Adherence to denosumab in the treatment of osteoporosis and its utilization in the Czech Republic. Curr Med Res Opin 2015;31:164553.

20. Kendler DL, Roux C, Benhamou CL, et al. Effects of denosumab on bone mineral density and bone turnover in postmenopausal women transitioning from alendronate therapy. J Bone Miner Res 2010;25:7281.

21. Karlsson L, Lundkvist J, Psachoulia E, Intorcia M, Strom O. Persistence with denosumab and persistence with oral bisphosphonates for the treatment of postmenopausal osteoporosis: a retrospective, observational study, and a meta-analysis. Osteoporos Int 2015;26:2401-11.

22. Warriner AH, Curtis JR. Adherence to osteoporosis treatments: room for improvement. Curr Opin Rheumatol 2009;21:356-62. 